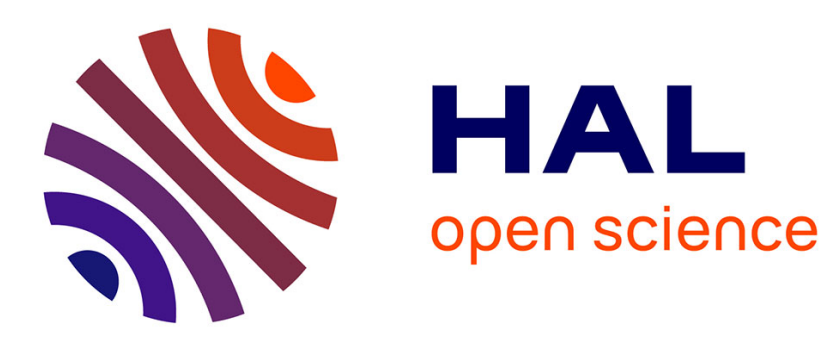

\title{
Multidimensional Calibration of the EXOGAM Segmented Clover
}

\author{
B. Rossé, O. Stezowski
}

\section{To cite this version:}

B. Rossé, O. Stezowski. Multidimensional Calibration of the EXOGAM Segmented Clover. Nuclear Instruments and Methods in Physics Research Section A: Accelerators, Spectrometers, Detectors and Associated Equipment, 2006, 565, pp.623-629. 10.1016/j.nima.2006.05.211 . in2p3-00112054

\section{HAL Id: in2p3-00112054 https://hal.in2p3.fr/in2p3-00112054}

Submitted on 7 Nov 2006

HAL is a multi-disciplinary open access archive for the deposit and dissemination of scientific research documents, whether they are published or not. The documents may come from teaching and research institutions in France or abroad, or from public or private research centers.
L'archive ouverte pluridisciplinaire HAL, est destinée au dépôt et à la diffusion de documents scientifiques de niveau recherche, publiés ou non, émanant des établissements d'enseignement et de recherche français ou étrangers, des laboratoires publics ou privés. 


\title{
Multidimensional Calibration of the EXOGAM Segmented Clover
}

\author{
B.Rossé ${ }^{a}$ and O.Stézowski \\ IPN Lyon, IN2P3/CNRS, Université Lyon-1, F-69622 Villeurbanne Cedex, France
}

\begin{abstract}
The EXOGAM clover detector consists of four high-purity germanium (HPGe) crystals in a common cryostat. Each individual crystal is electrically segmented into four regions. We propose a method based on a multidimensional fitting procedure to calibrate the energy from the segments. This method could be used for any kind of segmented germanium detector. Its application to the EXOGAM clover is discussed in details.
\end{abstract}

PACS: $29.30 . \mathrm{Kv} ; 29.40 . \mathrm{Gx} ; 07.05 . \mathrm{Kf}$

Keywords: Germanium detectors; Segmented detector; Gain matching

\section{Introduction}

EXOGAM is a compact, highly efficient and segmented germanium-detector array [1,2]. It is well suited for $\gamma$-ray spectroscopy at SPIRAL [3] which provides radioactive beams from ${ }^{6} \mathrm{He}$ to ${ }^{81} \mathrm{Kr}$ in an energy range of 1.5-25 A.MeV. The data set used for this work was collected during an experiment using such a radioactive beam, namely ${ }^{76} \mathrm{Kr}$ [4]. For this experiment, EXOGAM was composed of 11 segmented germanium clover detectors.

Because the design specifications of EXOGAM focused essentially on photopeak efficiency, large volume clovers are located close to the target point. The solid angle sustained by each crystal is thus rather large and represents a square surface of roughly $4 \times 4 \mathrm{~cm}^{2}$ at a distance of $11 \mathrm{~cm}$. In experimental situations when the recoil velocity of the emitting nuclei is large (typically a few percent of the speed of light), the Doppler effect considerably broadens photopeak events and destroys the resolving power of the whole array. A good mean to improve this is to use electric segmentation which significantly reduces the individual solid angle while preserving the efficiency. In EXOGAM, each clover consists of four independent crystals, closely packed together in the same housing, each one being electrically segmented in four regions. This represents a total of 176 different segments in our experiment. The charge from the whole crystal volume is collected with the central contact and gives an accurate measure of the energy of the incoming $\gamma$-rays while each individual segments are sensitive to only a fraction of the total volume. For a single crystal, it provides then a better localization of the interaction point when one segment fires. When two or more segments give non-zero energy (i.e. for Compton scattering events if one excludes pile-up), the determination of the first interaction point should be done using pulse shape analysis techniques, this development will be soon implemented in EXOGAM. Therefore, if the central contact gives the energy, the segment better defines the individual solid angle. Nevertheless, one may need to know precisely the segment energies e.g. in case the addback algorithm is able to disentangle, in a given crystal, between Compton scattering and pileup events. It is especially relevant to the next generation of $\gamma$-ray spectrometers in which the trajectories of photons are reconstructed. Indeed, $\gamma$-ray tracking algorithms are strongly

\footnotetext{
${ }^{a)}$ Corresponding author: IPN Lyon, Université Claude Bernard, 43 Boulevard du 11

Novembre 1918, F-69622 Villeurbanne, Cedex, France.

E-mail address: rosse@ipnl.in2p3.fr (B. Rossé)
} 
dependent of an accurate measurement of the segment energies. A general method to properly calibrate the latter is the subject of the present communication.

\section{Problem}

The central contacts have been calibrated with the "fuzzy logic" method [5] by using fullenergy peaks detected from ${ }^{60} \mathrm{Co}$ and ${ }^{152} \mathrm{Eu}$ sources. The associated electronics has been found extremely stable and only two sets of calibration coefficients are necessary for the whole experiment. As a consequence, the energy deposited in the whole crystal is always precisely measured. It will be hereafter denoted ECC (Energy of the Central Contact). The total energy ECC also equals the sum of the energies measured in the four individual segments. Assuming the ADC signal for the segment $i$ is $S_{i}$ and the calibration function is a polynomial equation of order 1, it follows:

$$
E C C=f\left(S_{1}, S_{2}, S_{3}, S_{4}\right)=\sum_{i=1}^{4} A_{i}^{s} S_{i}+B_{i}^{s}
$$

The different calibration coefficients $\left(A_{i}^{s}, B_{i}^{s}\right)$, obtained from "single hit" configurations, have been determined using the standard method with a ${ }^{152} \mathrm{Eu}$ source. In our data set, for about $80 \%$ of the events, only one segment fired. Thus, photopeaks appear clearly in the spectra and could be used to calibrate the segments. Considering Eq. (1), the segment calibration can be checked by displaying, for instance, the ECC quantities as a function of the reconstructed energy $f\left(S_{1}, S_{2}, S_{3}, S_{4}\right)$. If the calibration is correct, the data points are located on the first diagonal. Such a plot is represented in Fig. 1A, for one particular crystal, with a restricted energy range around the ${ }^{152} \mathrm{Eu} 1408.0 \mathrm{keV} \gamma$-ray line.
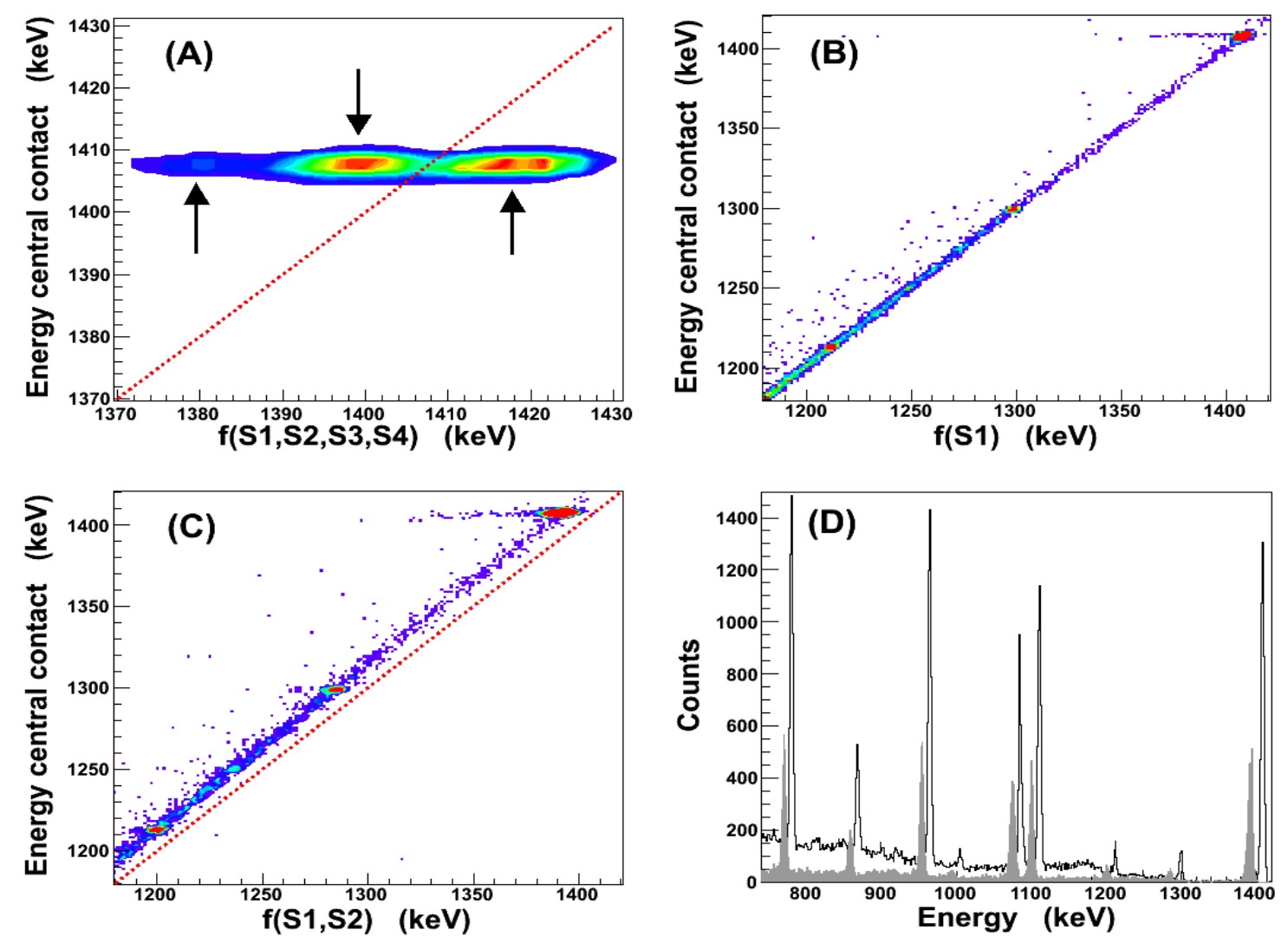
Figure 1: In A, B and C, correlation matrix representing the central contact energy ECC as a function of the reconstructed energy from the segment signals. The calibration coefficients are obtained with the standard method. In $B$ and $C$, respectively one and two segments fired. The two projections of the correlation matrix depicted in $\mathrm{C}$ (along vertical and horizontal axis) are compared in $D$. Filled in white is the central contact energy and in gray the reconstructed segment energy.

While only one peak is expected, a surprising structure appears with three different components pointed out by arrows in Fig. 1A. The same structure is observed for all the known $\gamma$-ray energies of the ${ }^{152} \mathrm{Eu}$ source. To understand such a structure, Fig. 1B and Fig. 1C show similar plots for different classes of events and on a larger energy range. In Fig. 1B, "single hit" events are selected and Eq.(1) reads:

$$
E C C=f\left(S_{1}\right)=A_{1}^{s} S_{1}+B_{1}^{s}
$$

Almost all the points are scattered along the first diagonal attesting to the correct calibration of this particular segment. Few events (less than 0.5\%) corresponding to central contact energy around the ${ }^{152} \mathrm{Eu} 1408.0 \mathrm{keV} \gamma$-ray line appear in a short horizontal tail. For these events the segment energy does not match the central contact one; a small fraction, from a few $\mathrm{keV}$ up to $50 \mathrm{keV}$, is missing. To determine whether or not a segment fired, a software threshold was applied on the ADC values corresponding to the energy. From Fig. 1B it appears that the threshold was slightly higher than the pedestal which explains why, for some events, the segment energy does not match ECC.

In Fig. 1C, "double hits" events are considered and Eq. (1) reads:

$$
E C C=f\left(S_{1}, S_{2}\right)=A_{1}^{s} S_{1}+B_{1}^{s}+A_{2}^{s} S_{2}+B_{2}^{s}
$$

The clear discrepancy observed proves that the calibration coefficients are wrong: the 1408.0 $\mathrm{keV}$ peak is off by about $20 \mathrm{keV}$. It is worth noting that this discrepancy is not a global simple shift but is energy dependent as demonstrated in Fig. 1D. The first spectrum (filled in white) represents the central contact energy spectrum ECC and the second one (filled in gray) corresponds to the reconstructed energy from "double hits" events. While the $778.9 \mathrm{keV}$ peaks for the two spectra overlap, the difference increases with energy and the $1408.0 \mathrm{keV}$ peaks are clearly separated.

Coming back to Fig. 1A, it appears that the three peaks, from right to left, correspond to events in which respectively one, two and three segments fired. This effect comes from electronic crosstalks that have been revealed by studying position-dependent response of different segmented germanium detectors [6-8]. Two types of crosstalks have been established. The first so-called "baseline" crosstalk gives an apparent net charge in a noncollecting segment. Such "baseline" shift is position-independent and can be compensated by adding a fraction of the collecting segment signal. The second so-called "derivative" crosstalk is position-dependent and can be compensated by subtracting a fraction of the derivative of the collecting segment signal. For our experiment, only ADC values of the signals are recorded while the proposed correction applies on time-dependent signal: what we observe are integrated values of the crosstalks. Since the "baseline" crosstalk is position-independent, it should be the one that contributes much to ADC signals. The differences between the central contact energy and the reconstructed one, in case of "double hits" events (about 1.4\% at $1408.0 \mathrm{keV}$ ), indeed compare with the measured "baseline" crosstalk [7].

To correct for "multiple hits" effects and to properly calibrate the segment spectra, one set of coefficients has to be determined whatever the number of fired segments. The standard 
method can not be applied to achieve this goal because there are no peaks in the segment spectrum in the case where more than one segment fired. We propose to use the central contact energy ECC as already done by $\mathrm{Hu}$ et al. [9] to calibrate the outer contact energy signals. While $\mathrm{Hu}$ et al. restricted their study to "single hit" configurations, we propose in this paper to extend the calibration to "multiple hits" configurations. In the next section, the method is presented using the simulations performed to validate the calibration procedure.

\section{Calibration method and simulations}

In this work, we focus on "single hit" (only one segment fired) and "double hits" (two segments amongst four fired) configurations because, in our data set, the number of three- and four-fold events is not statistically significant. However, we emphasize that the proposed calibration method can be easily extended to three and four hits configurations.

As already mentioned above, the central contact energy is used to calibrate the segments. In the "single hit" configurations, the fitting procedure is applied with the following onedimensional function

$$
f\left(S_{i}\right)=A_{i}^{s} S_{i}+B_{i}^{s}
$$

and the coefficients $\left(A_{i}^{s}, B_{i}^{s}\right)$ for any segment $i$ are determined by

$$
f\left(S_{i}\right)=E C C
$$

For the "double hits" configurations, the fitting procedure is applied with the two-dimensional function

$$
f\left(S_{i}, S_{j}\right)=A_{i}^{d} S_{i}+A_{i}^{d} S_{j}+B_{i j}^{d}
$$

where the coefficients $\left(A_{i}^{d}, A_{i}^{d}, B_{i j}^{d}\right)$, related to "double hits" events, for any $i j$ combination are determined by

$$
f\left(S_{i}, S_{j}\right)=E C C
$$

Before applied to experimental data, the above procedure has been extensively tested by means of simulations. The calibration coefficients A and B are generated randomly within reasonable limits for our data set. Once these coefficients are fixed, a set of $\mathrm{N}$ events is randomly produced. For the central contact energy, the experimental ${ }^{152} \mathrm{Eu}$ spectrum is used as a generator. The choice of the input spectrum obviously has no impact on the results, and only the correlation between the central contact and the segments is mandatory. Thus, it does not matter whether the incoming $\gamma$-rays correspond to photopeaks or background. Once the central contact energy is established, it is degraded before being assigned to a given segment (in the case of a "single hit" event) to account for the experimental resolution. Then, the simulated segment energy is randomly sampled on a Gaussian function. Its mean value equals to the ECC value and its full-width at half-maximum (FWHM) depends linearly on the energy: it has been set to $3.0 \mathrm{keV}$ and $3.5 \mathrm{keV}$ for respectively $100 \mathrm{keV}$ and $1000 \mathrm{keV}$. The corresponding uncalibrated signal is then assigned by inverting Eq.(4). For "double hits" configurations, the central contact energy is uniformly distributed over the two segments. 
Such a simple approach is nevertheless realistic since we observe, in our data, a uniform distribution except at very low energy because of threshold effects. Both simulated energies are finally degraded independently as described for "single hit" configurations.
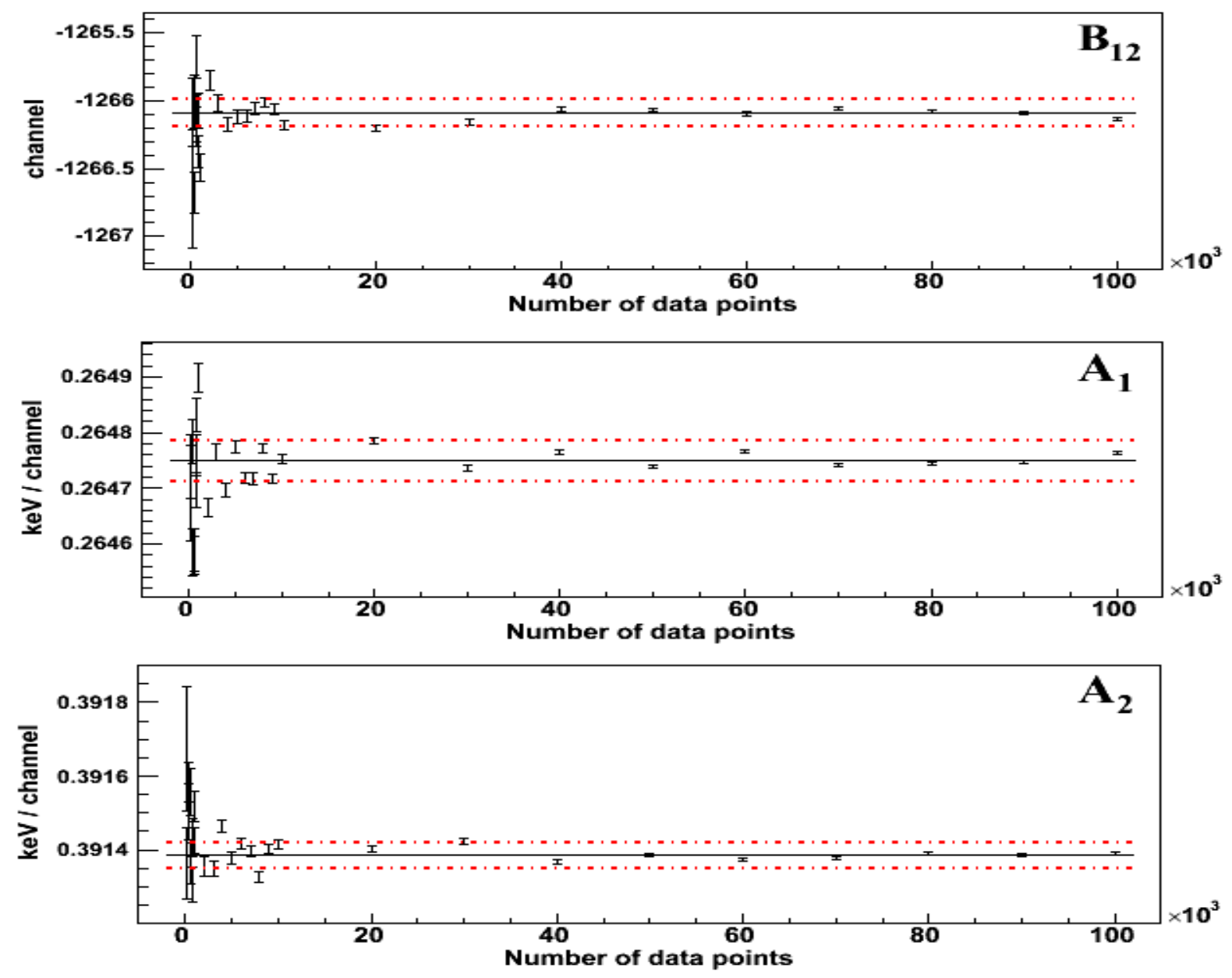

Figure 2: Comparison of the coefficients from Eq. (6) for the "double hits" configuration obtained by the fitting procedure (points) as a function of the number of events in the simulated data set. Full lines represent the input value while dashed dotted lines materialize the binning limit.

Several fitting procedures have been tested with the simulated data sets and the TLinearFitter implementation provided by the ROOT framework [10] has been selected. The fitting process is extremely fast since the implementation is optimized for the type of calibration functions considered in this work. It is important to minimize the processing time because a set of parameters must be determined for any combination of one, two, three and four segments that are participating to the total energy deposited in a given crystal. The accuracy of the fitting procedure is ascertained in Fig. 2 for a particular "double hits" configuration. The parameters resulting from the fit (points on the pictures) are compared to the input values (horizontal full lines) for an increasing number of simulated data points ranging from $10^{2}$ to $10^{5}$. With a minimum of $10^{4}$ points, the results for the three coefficients are within the binning limit materialized by the dashed dotted lines, representing an excellent precision (less than 0.366 $\mathrm{keV}$ ). Thus, it allows the monitoring of extremely weak gain drift. Applied to our data set, with a sampling every 50000 events, the segment contacts have been found much less stable than the central contact. 
At last, the resolution achieved in the case of "double hits" events have been compared to "single hit" configurations. In the former case, the FWHM is larger by about $20 \%$ and $25 \%$ for the $344.3 \mathrm{keV}$ and the $1408.0 \mathrm{keV}{ }^{152} \mathrm{Eu} \gamma$-rays, respectively. As shown in the next section, this result will be used to check precisely the calibration for experimental data.

\section{Application to experimental data}

The most accurate way to check the calibration is to determine the position of all the peaks in the calibrated spectrum and to compare them with tabulated values. The results are summarized in Table 1 in which the position and the resolution (FWHM) of three different peaks of the ${ }^{152} \mathrm{Eu}$ source for a particular crystal are indicated.

\begin{tabular}{|c|c|c|c|}
\hline tabulated & $344.3 \mathrm{keV}$ & $964.1 \mathrm{keV}$ & $1408.0 \mathrm{keV}$ \\
\hline "single hit" & $344.4 \pm 0.2(3.5) \mathrm{keV}$ & $964.1 \pm 0.2(3.6) \mathrm{keV}$ & $1407.9 \pm 0.3(3.7) \mathrm{keV}$ \\
\hline "double hits" & $343.7 \pm 0.2(6.6) \mathrm{keV}$ & $964.4 \pm 0.2(5.1) \mathrm{keV}$ & $1406.4 \pm 0.3(5.3) \mathrm{keV}$ \\
\hline
\end{tabular}

Table 1: Position of three ${ }^{152} \mathrm{Eu}$ peaks after calibration for "single hit" and "double hits" configurations. The values in parenthesis correspond to the associated FWHM.

For "single hit" configurations, the peak positions match with the expected values within the errors. This confirms that the coefficients are correctly calculated and justifies the choice of a one order polynomial.

The situation is slightly different for "double hits" configurations. The calibration obtained with the approach outlined in section 3 is much better as compared to the one resulting from the application of the coefficients coming from "single hit" configurations which leads, as mentioned in section 2, to a $1408.0 \mathrm{keV}$ peak off by about $20 \mathrm{keV}$. To illustrate this point, some coefficients are listed in Table 2 for two particular segments as well as reconstructed energies that should match the $344.3 \mathrm{keV}$ and $1408.0 \mathrm{keV} \gamma$-ray lines.

\begin{tabular}{|c|c|c|c|}
\hline \multicolumn{2}{|c|}{ Calibration from "single hit" } & \multicolumn{2}{c|}{ Calibration from "double hits" } \\
\hline$A_{1}^{s}$ & 0.4485 & $A_{1}^{d}$ & 0.4410 \\
\hline$A_{2}^{s}$ & 0.4366 & $A_{2}^{d}$ & 0.4516 \\
\hline$B_{1}^{s}+B_{2}^{s}$ & -378.6 & $B_{12}^{d}$ & -380.4 \\
\hline \multirow{2}{*}{$A_{1}^{s} S_{1}+B_{1}^{s}+A_{2}^{s} S_{2}+B_{2}^{s}$} & $(336.5 \pm 0.2) \mathrm{keV}$ & \multirow{2}{*}{$A_{1}^{d} S_{1}+A_{2}^{d} S_{2}+B_{12}^{d}$} & $(343.7 \pm 0.2) \mathrm{keV}$ \\
\cline { 2 - 4 } & $(1392.5 \pm 0.3) \mathrm{keV}$ & & $(1406.4 \pm 0.3) \mathrm{keV}$ \\
\hline
\end{tabular}

Table 2 : "Single hit" and "double hits" gain and offset coefficients for 2 particular segments. Positions after application of the coefficients to "double hits" events for the ${ }^{152} \mathrm{Eu} 344.3 \mathrm{keV}$ and $1408.0 \mathrm{keV} \gamma$-ray lines.

Table 2 proves again that the coefficients for "double hits" configurations can not be deduced simply from the "single hit" ones: gain and offset are both significantly different. Applying the new coefficients for "double hits" events, in a correlation plot similar to the one of Fig. $1 \mathrm{C}$, the data points are well located, as expected, on the first diagonal. However, even if the calibration is satisfying, it is not as good as what obtained for "single hit" events. From Table 
1 (line 2), it is seen that the peak positions are slightly different from what is expected. Looking closer to our data, we found that the linearity is much better if the energy deposited in both segments exceeds about $250 \mathrm{keV}$. Moreover, the FWHM are 20 to $25 \%$ larger than what is expected from "single hit" events in the simulations of section 3.

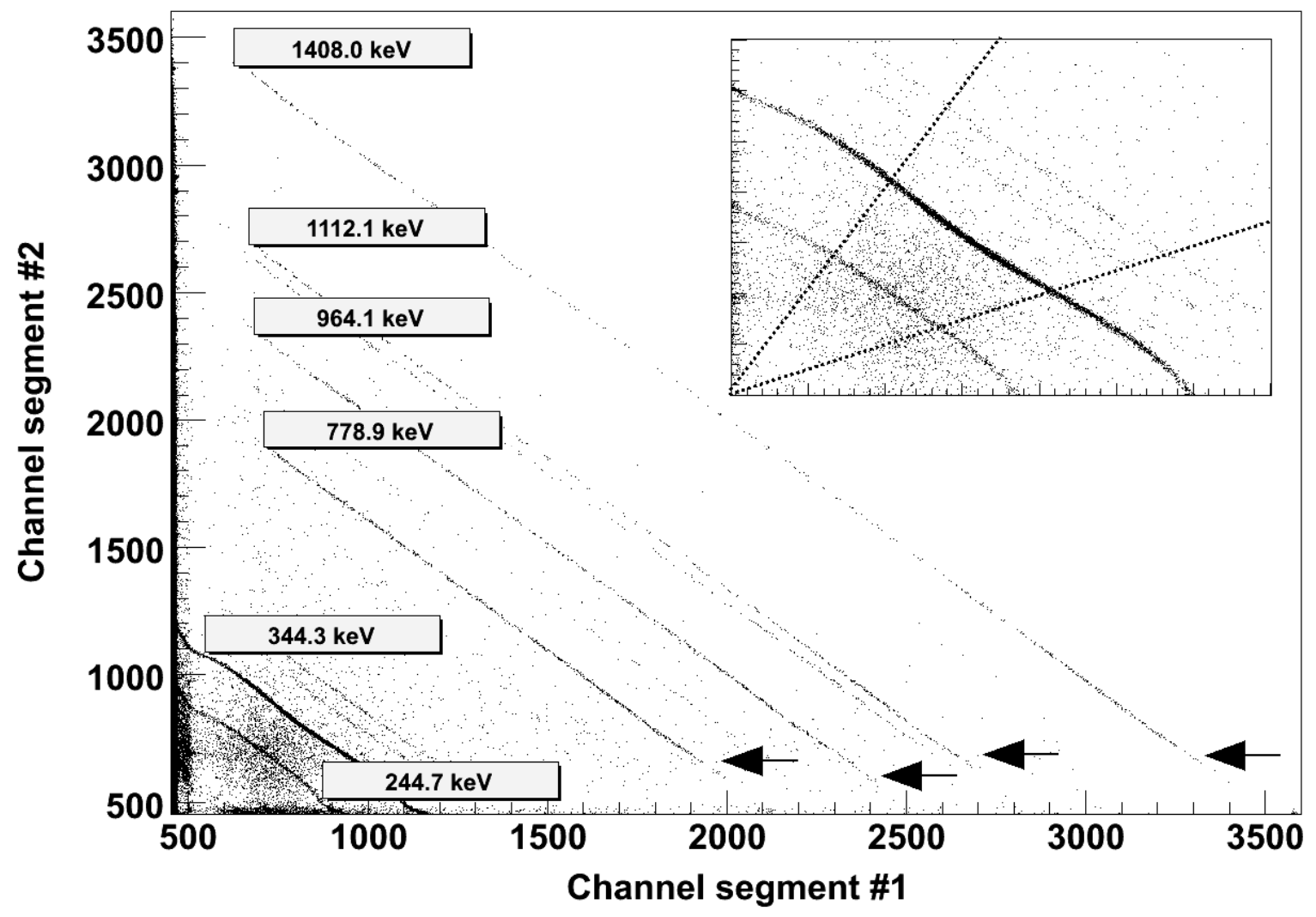

Figure 3: Correlation matrix that represents the uncalibrated energy signal of one segment as a function of another one for a given crystal. The insert is a zoom on the low part of the matrix. The arrows indicate the slight breaking of the slope that appears as soon as the energy collected in segment $\# 2$ is low. The dotted lines in the insert delimit the symmetric and asymmetric partitions (see the text for explanations).

These differences could be understood by looking at the correlation histogram displayed in Fig. 3 where the uncalibrated segment signal $S_{2}$ is presented as a function of $S_{1}$. The lines nearly perpendicular to the first diagonal, materialize Compton scattering events which give photopeak energies when added back. The different lines correspond to the most intense ${ }^{152} \mathrm{Eu}$ $\gamma$-rays. The peak at $121.784 \mathrm{keV}$ does not appear in this histogram since the photoelectric effect (i.e. "single hit") dominates Compton scattering at this energy.

At higher energies $(778.9 \mathrm{keV}, 964.1 \mathrm{keV}, 1112.1 \mathrm{keV}$ and $1408.0 \mathrm{keV})$ the linearity is well established except as soon as the energy deposited in one segment is small. It is materialized by a slight break in the slope as pointed out by arrows in Fig. 3. For the two smaller peaks ( $244.7 \mathrm{keV}$ and $344.3 \mathrm{keV}$ ), the linearity is less obvious, especially for the $344.3 \mathrm{keV}$ peak, as shown in insert. However, non-linear effects seem stronger when one of the segment carries less energy than the other one.

To a better understanding of the link between linearity and small deposited energy, the data set has been divided in two classes of events: those for which the total energy of the crystal ECC is equally distributed over two segments (denoted symmetric partition) and those for which the distribution is unbalanced (asymmetric partition). Such a selection separates the 
matrix displayed in Fig. 3 in two parts materialized in the insert by two dotted lines: the symmetric partition corresponds to points located inside the dashed lines around the first diagonal, while in asymmetric partition, the points are outside. The results are presented in Fig. 4 for an energy range around the $344.3 \mathrm{keV} \gamma$-ray where non-linear effects are more pronounced. It appears that the reconstructed $344.3 \mathrm{keV}$ peak has a fine structure depending on the event class previously defined. The FWHM for each individual class of events is reduced and approaches, for symmetric partitions, what expected from the simulation. The exact origin of the non-linear effects is difficult to determine with our data set. Looking meticulously at all correlation histograms similar to the one in Fig. 3, there are no significant differences at low energy (244.7 keV and $344.3 \mathrm{keV})$. However, at higher energies (778.9 $\mathrm{keV}, 964.1 \mathrm{keV}, 1112.1 \mathrm{keV}$ and $1408.0 \mathrm{keV}$ ), the slope's breaking seems reduced (the statistics is very poor) for diagonal, non-neighbouring, contacts. Low energy $\gamma$-rays interact in the germanium detector mainly near the front, while higher energy photons interact deeper. In case an interaction occurs at the frontier between two contacts, an induced signal is observed on the non-collecting segment up to $10 \%$ the amplitude of the net-charge-collecting signal. Because of its geometry, near the front of the EXOGAM clover, all segments can influence each other while it is not possible at the back for diagonal contacts because of the hole in the crystal. Then what we observe might be consistent with real transient signals as origin of the non-linear effects. From the slope's breaking, the extra induced signal has been roughly estimated (about 10\%) giving another positive hints. However, electronic crosstalks can not be excluded and more dedicated studies, based on position-dependent measurements, are needed to settle this point.

Thus, while "single hit" events can be calibrated with a linear function, it is necessary to take into account non-linear effects, especially at low energy, to achieve a precise calibration for "double hits" configurations. This can be done by using a more complex calibration function as proposed for instance by Pattabiraman et al ([11] and references within). For our experiment, this level of precision was not needed since the segments were used only to reduce the Doppler broadening.

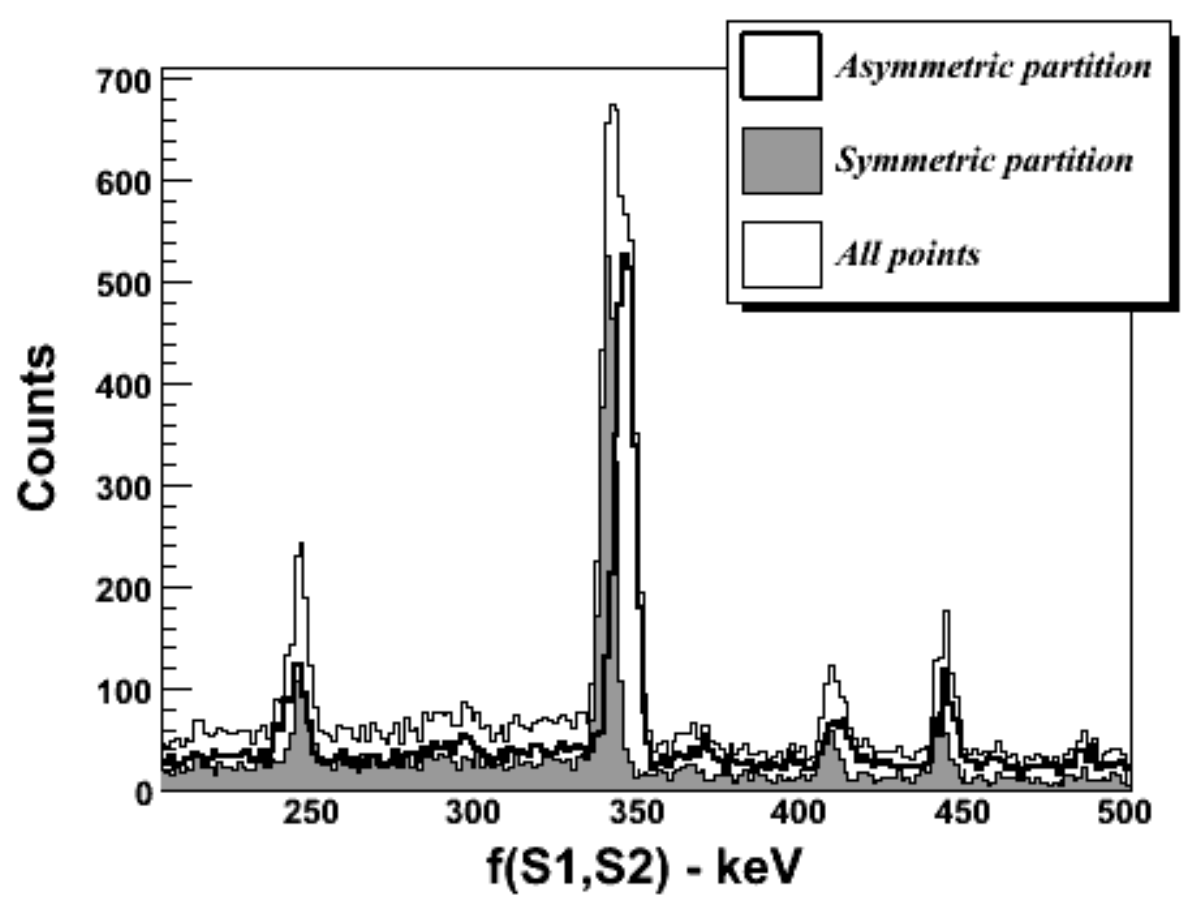


Figure 4: Part of the calibrated energy spectrum for a particular "double hits" configuration. The spectrum built with all events is composed of two classes of events depending on how the central contact energy is distributed over the two segments fired.

\section{Summary}

We have demonstrated that the calibration coefficients of the segmented EXOGAM clover detectors depend not only on the number of segments fired but also on which ones. Thus, fifteen (four for "single hit", six for "double hits", four for "three hits" and one for "four hits" configurations) sets of coefficients are required to properly calibrate a crystal. The proposed calibration method, based on the use of the central contact energy, could be applied whatever the configuration and to any kind of electrically segmented germanium detector.

While, for EXOGAM clovers, a linear calibration gives excellent results for "single hit" configurations, strong non-linear effects have been observed for "double hits" configurations, especially in the case where one of the segment collects a low energy.

\section{Acknowledgement}

The authors would like to thank all the people involved in the E404aS experiment for their essential help. Thanks are due also to G. de France for enlightening discussions.

\section{References}

[1] F. Azaiez, Nucl. Phys. A 654 (1999) 1003c.

[2] J. Simpson, F. Azaiez, G. de France, J. Fouan, J. Gerl, R. Julin, W. Korten, P.J. Nolan, B.M. Nyakó, G. Sletten, P.M. Walker, and the EXOGAM Collaboration, Heavy Ion Phys. 11 (2000) 159.

[3] M. Lieuvin, Proceedings of the sixth European Particle Accelerator Conference, Stockholm, Sweden, 22-26 June 1998, p. 68.

[4] B. Rossé, Proceedings of the International conference on frontiers in Nuclear Structure, astrophysics and reactions, Kos, Grece, 12-17 September 2005, to be published.

[5] O. Stézowski, A. Astier, A. Prévost, A.G. Smith, R. Wall, Nucl. Instr. And Meth. A 488 (2002) 314.

[6] K. Vetter, A. Kuhn, I.Y. Lee, R.M. Clark, M. Cromaz, M.A. Deleplanque, R.M. Diamond, P. Fallon, G.J. Lane, A.O. Macchiavelli, M.R. Maier, F.S. Stephens, C.E. Svensson, H. Yaver, Nucl. Instr. And Meth. A 452 (2000) 105.

[7] A. Boston, private communication.

[8] C.E. Svensson et al, Nucl. Instr. And Meth. A 540 (2005) 348.

[9] Z. Hu, T. Glasmacher, W.F. Mueller, I. Wiedenhöver, Nucl. Instr. and Meth. A 482 (2002) 715.

[10] R. Brun, F. Rademakers, ROOT-an object oriented data analysis framework, Proceeding of the AIHENP'96 Workshop, Lausanne, September 1996, Nucl. Instr. and Meth. A 389 (1997) 81.

[11] N.S. Pattabiraman, S.N. Chintalapudi, S.S. Ghugre, Nucl. Instr. and Meth. A 526 (2004) 432. 